\title{
Zähmt ein Antikonvulsivum die Kopfschmerzattacken?
}

Fragestellung: Ist Gabapentin in der Prophylaxe der Migräne wirksam?

Hintergrund: Sinn der medikamentösen Migräneprophylaxe ist eine Reduktion der Häufigkeit, Intensität und Dauer der Migräneattacken. Empfohlene Prophylaktika umfassen Betablocker wie Propranolol, Metoprolol oder Bisoprolol, den Kalziumantagonisten Flunarizin und die Antikonvulsiva Valproinsäure und Topiramat. Es gibt eine Reihe kleinerer offener Studien, die eine Wirksamkeit von Gabapentin in der Migräneprophylaxe zeigten. Zwei placebokontrollierte Studien aus den Jahren 2000 und 2001 zeigten eine Wirksamkeit von Gabapentin, wobei aber beide Studien schwerwiegende methodische Mängel hatten. Daher sollte jetzt in einer Dosisfindungsstudie nochmals untersucht werden, ob Gabapentin zur Migräneprophylaxe wirksam ist.

Patienten und Methodik: Eingeschlossen wurden Migränepatienten mit oder ohne Aura mit mindestens drei Migräneattacken pro Monat. Ausgeschlossen wurden Patienten mit chronischer Migräne. Nach einer sechswöchigen Baselinephase wurden die Patienten randomisiert und dann über 20 Wochen behandelt. In den ersten fünf Wochen wurde Gabapentin eindosiert, dann erfolgte über zwölf Wochen eine gleichbleibende Behandlung. In den letzten drei Wochen wurde die Studienmedikation wieder ausgeschlichen. Untersucht wurden vier Dosierungen von Gabapentin, nämlich $1.200 \mathrm{mg} / \mathrm{d}, 1.800 \mathrm{mg} / \mathrm{d}, 2.400$ $\mathrm{mg} / \mathrm{d}$ und $3.000 \mathrm{mg} / \mathrm{d}$ und mit Placebo verglichen. Die Randomisierung erfolgte im Verhältnis $2: 1: 2: 2: 1$. Der primäre Endpunkt war die Änderung der Migränetage in den letzten vier Wochen der Behandlung bevor die Dosisreduktion erfolgte.

Ergebnisse: Die Patienten waren im Mittel zwischen 38 und 41 Jahre alt, 74-87\% waren Frauen. Die mittlere Zahl der Migräneattacken lag bei 9 bis 9,3 pro Monat. Die Zahl der Patienten in den einzelnen Behandlungsgruppen betrug 129 für Placebo, 67 für $1.200 \mathrm{mg}$, 134 für $1.800 \mathrm{mg}, 133$ für $2.400 \mathrm{mg}$ und 62 für $3.000 \mathrm{mg}$ Gabapentin. Ein Viertel bis ein Drittel der Patienten brach die Behandlung vorzeitig ab. Für keine der GabapentinDosierungen ergab sich eine signifikante Überlegenheit gegenüber Placebo für den primären Endpunkt. Auch für alle anderen Endpunkte wie beispielsweise eine über $50 \%$ ige Reduktion der Migränetage, der Einnahme der Akutmedikation, der Dau-

Silberstein S, Goode-Sellers S, Twomey C et al. Randomized, double-blind, placebo-controlled, phase II trial of gabapentin enacarbil for migraine prophylaxis. Cephalalgia. 2013; 33: $101-11$ er der Migräneattacken, der Schwere der Migräneattacken beziehungsweise für die Endpunkte Übelkeit, Erbrechen, Licht- und Lärmempfindlichkeit ergab sich ebenfalls kein signifikanter Unterschied zwischen Gabapentin und Placebo. Erwartungsgemäß

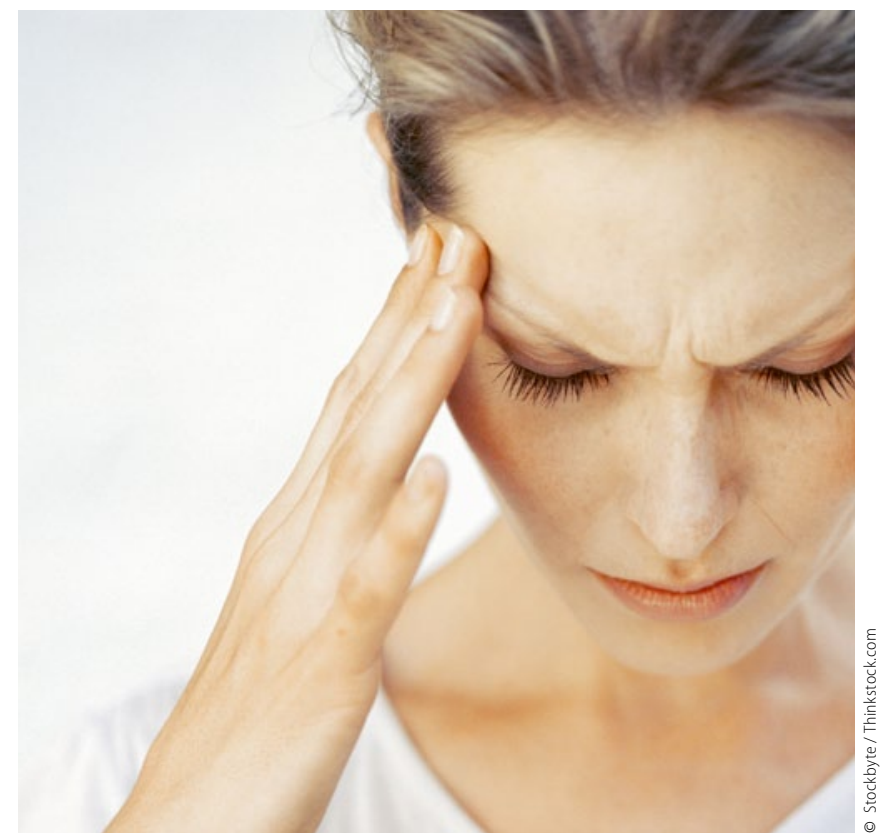

Die deutschen Migräneleitlinien waren in punkto Gabapentin schon immer zurückhaltend. Nun kann als gesichert gelten, dass das Antikonvulsivum zur Migräneprophylaxe nichts bringt.

waren Nebenwirkungen wie Schwindel, Müdigkeit und Benommenheit häufiger bei Gabapentin als bei Placebo.

Schlussfolgerung: Gabapentin ist zur Migräneprophylaxe nicht wirksam.

\section{- Kommentar von Hans-Christoph Diener, Essen Die Diskrepanz ist aufgelöst}

Mit dieser gut durchgeführten großen Dosisfindungsstudie ist nun eine Diskrepanz zwischen den US-amerikanischen und den deutschen Leitlinien zur Migräneprophylaxe aufgelöst. In den deutschen Leitlinien hatten wir uns nie dazu durchringen können, Gabapentin zu empfehlen, da wir der Meinung waren, dass die beiden früheren placebokontrollierten Studien, die die Grundlage für die Empfehlung in den USA bildeten, methodisch so schlecht waren, dass eine Wirksamkeit nicht zu belegen war. Außerdem gab es immer wieder Gerüchte, dass es auch weitere unpublizierte Studien zu Gabapentin gab, was nahe legte, dass diese Studien negativ waren. In der vorliegenden Studie wurde Gabapentin enacarbil verwendet, ein Prodrug für Gabapentin, bei dem aber nicht anzunehmen ist, dass sich seine Wirksamkeit von Gabapentin unterscheidet. 